\title{
Algoritmo de Seguimento do Ponto de Máxima Potência para Sistemas Fotovoltaicos considerando Minimização de Erro e Derivada da Potência
}

\author{
G. LOPES FILHO ${ }^{1 *}$, R. A. P. FRANCO ${ }^{2}$ e F. H. T. VIEIRA ${ }^{3}$
}

Recebido em 16 de abril de 2019 / Aceito em 15 de novembro de 2019

\begin{abstract}
RESUMO. Neste trabalho, é proposto um novo algoritmo de seguimento de ponto de máxima potência (MPPT, do inglês Maximum Power Point Tracking) para sistemas fotovoltaicos. O sistema considerado consiste de um painel fotovoltaico que fornece energia a uma bateria por intermédio de um conversor cc-cc do tipo buck (abaixador de tensão). São realizadas simulações computacionais para se comparar o desempenho dos algoritmos de MPPT, analisando os comportamentos da tensão e potência na saída do painel fotovoltaico. Foram considerados dois cenários para avaliação do desempenho do algoritmo de MPPT proposto: com irradiância e temperatura constantes, e utilizando dados reais. Os resultados das simulações comprovam que o algoritmo proposto provê melhor desempenho para o sistema fotovoltaico do que os principais algoritmos de MPPT existentes na literatura.
\end{abstract}

Palavras-chave: algoritmo MPPT, energia solar, painel fotovoltaico.

\section{INTRODUÇÃO}

A energia solar fotovoltaica é uma energia limpa e renovável, e tem aumentado sua popularidade nos últimos anos [6]. O conjunto de células fotovoltaicas formam um painel fotovoltaico. A geração de energia solar fotovoltaica está relacionada diretamente com o nível de irradiância incidente no painel fotovoltaico [13].

Os painéis fotovoltaicos dificilmente são conectados diretamente a uma carga [2]. No caso da alimentação de uma carga de corrente contínua (cc), esta conexão é feita por meio de um circuito intermediário, geralmente um conversor cc-cc. O conversor cc-cc necessita de um sinal de referência, denominado ciclo de trabalho (duty cycle), que determina o ganho de tensão da saída

\footnotetext{
*Autor Correspondente: Gilberto Lopes Filho - E-mail: gilberto.lopes@ufg.br

${ }^{1}$ Escola de Engenharia Elétrica, Mecânica e de Computação (EMC), Universidade Federal de Goiás (UFG). E-mail: gilberto.lopes@ufg.br https://orcid.org/0000-0003-4850-6978

${ }^{2}$ Escola de Engenharia Elétrica, Mecânica e de Computação (EMC), Universidade Federal de Goiás (UFG). E-mail: ricardofranco@ufg.br https://orcid.org/0000-0001-7169-3367

${ }^{3}$ Escola de Engenharia Elétrica, Mecânica e de Computação (EMC), Universidade Federal de Goiás (UFG). E-mail: flavio_vieira@ufg.br https://orcid.org/0000-0003-3572-4036
} 
do conversor. Este sinal de referência, quando se trata de painéis fotovoltaicos, é determinado por um algoritmo que rastreia a máxima potência que o painel pode fornecer. Usualmente estes algoritmos são denominados de algoritmos de Seguimento do Ponto de Máxima Potência ( Maximum Power Point Tracking - MPPT).

Em [10], é analisado o desempenho de um painel fotovoltaico conectado a um conversor cc-cc do tipo elevador de tensão (boost), com um controlador fuzzy que ajusta a tensão de máxima potência. Em [1], os autores apresentam uma abordagem de rastreamento do ponto de máxima potência baseada no método Perturbe e Observe, medindo-se apenas a corrente de saída do painel. Em [14], é apresentada uma comparação entre alguns algoritmos de MPTT quando os painéis são conectados a uma carga qualquer com tensão constante. Em [12], os autores realizam uma análise de vários algoritmos MPPT distintos. Os trabalhos analisados se diferem entre si em aspectos como complexidade, custo de implementação, eficiência, sensores utilizados, acurácia no seguimento do ponto de máxima potência quando a temperatura e irradiância variam.

Neste trabalho, diferente dos anteriormente mencionados, propõe-se que o ponto de operação do painel fotovoltaico seja ajustado de acordo com o erro em relação à máxima potência que ele pode fornecer (calculado por meio de uma expressão com origem na derivada da curva $P$ $V$ em relação à tensão) e à derivada da potência no tempo. Mais especificamente, propõe-se um algoritmo de rastreamento do ponto de máxima potência tendo como base o algoritmo de condutância incremental [4] mas que considera a convergência do erro de estimação de máxima potência e a derivada da potência no tempo. Para o cenário de aplicação do algoritmo proposto, considera-se um painel fotovoltaico alimentando uma bateria por meio de um conversor cc-cc do tipo buck. São analisados os desempenhos de quatro algoritmos de rastreamento do ponto de máxima potência em comparação ao algoritmo proposto neste trabalho. As simulações são realizadas no ambiente computacional MATLAB/SIMULINK [9].

O artigo está organizado da seguinte forma: a Seção 2 apresenta a modelagem do painel fotovoltaico utilizada na simulação; a Seção 3 apresenta os principais algoritmos MPPT conhecidos na literatura; a Seção 4 apresenta o algoritmo de MPPT proposto neste trabalho, a metodologia empregada para avaliação de desempenho dos algoritmos considerados; os resultados das simulações dos algoritmos MPPT e as comparações de desempenho são mostrados na Seção 5; a Seção 6 apresenta as conclusões do trabalho.

\section{MODELO DO PAINEL FOTOVOLTAICO}

O modelo do painel fotovoltaico com um diodo (Single Diode Model - SDM) é o modelo escolhido para este trabalho. Esta escolha se justifica pois o equacionamento do modelo de um diodo provê uma boa representação do painel fotovoltaico [13]. O circuito elétrico do SDM é apresentado na Figura 1. 


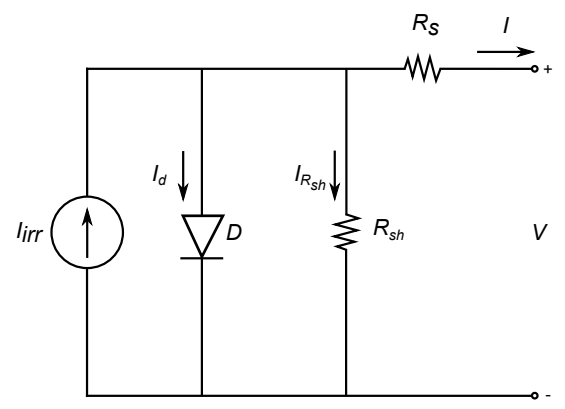

Figura 1: Circuito elétrico que representa o painel fotovoltaico (Modelo com um Diodo).

Aplicando a Lei de Kirchhoff das Correntes no circuito elétrico que representa o painel fotovoltaico, apresentado na Figura 1, e realizando algumas manipulações algébricas, obtém-se a equação característica do modelo SDM:

$$
I=I_{i r r}-I_{0}\left[e^{\left(\frac{V+I R_{s}}{N_{s} n V_{t}}\right)}-1\right]-\frac{V+I R_{s}}{R_{s h}}
$$

onde $I_{i r r}$ é corrente fotogerada; $I_{0}$ é a corrente de saturação reversa do diodo no escuro; $I_{R_{s h}}$ é a corrente em $R_{s h} ; N_{s}$ corresponde ao número de células em série que compõem o módulo fotovoltaico; $n$ é o fator de idealidade do diodo; $R_{s}$ e $R_{s h}$ são as resistências em série e paralela, respectivamente; $V$ e $I$ são os valores de tensão e corrente de saída do painel, respectivamente, e $V_{t}$ corresponde à tensão térmica $(\mathrm{V})$

Para realizar as simulações deste trabalho, foi escolhido o painel fotovoltaico Kyocera KC200GT [5]. As características elétricas deste painel nas Condições Padrões de Teste (Standart Test Conditions) são: $I_{m p}=7,61 \mathrm{~A}, V_{m p}=26,3 \mathrm{~V}, P_{\max }=200 \mathrm{~W}, I_{s c}=8,21 \mathrm{~A}, V_{o c}=32,90 \mathrm{~V}, N_{s}=54$.

A curva de potência do painel fotovoltaico em função da tensão pode ser traçada para vários valores de irradiâncias, conforme é mostrado na Figura 2. É desejável que o painel sempre opere na máxima potência, independente das condições ambientais, como temperatura e irradiância. Caso um algoritmo de MPPT não seja utilizado, o painel fotovoltaico operaria em um ponto da curva P-V abaixo do valor máximo, diminuindo valor da energia gerada.

\section{ALGORITMOS DE SEGUIMENTO DE PONTO DE POTÊNCIA MÁXIMA}

A potência fornecida pelo painel fotovoltaico depende das condições ambientais (irradiância e temperatura) e da carga nele conectada. Assim, a impossibilidade de escolher arbitrariamente as condições climáticas faz com que todo o controle de potência seja feito por meio da carga vista pelo painel. Isso é realizado por meio de um conversor cc-cc. Para controlar a potência fornecida do painel para a carga, ajusta-se o ciclo de trabalho do conversor conforme desejado. A busca do ciclo de trabalho do conversor cc-cc que fornece a maior potência que o painel pode prover, para uma dada irradiância e temperatura, geralmente é realizada por meio de Algoritmos de MPPT. 


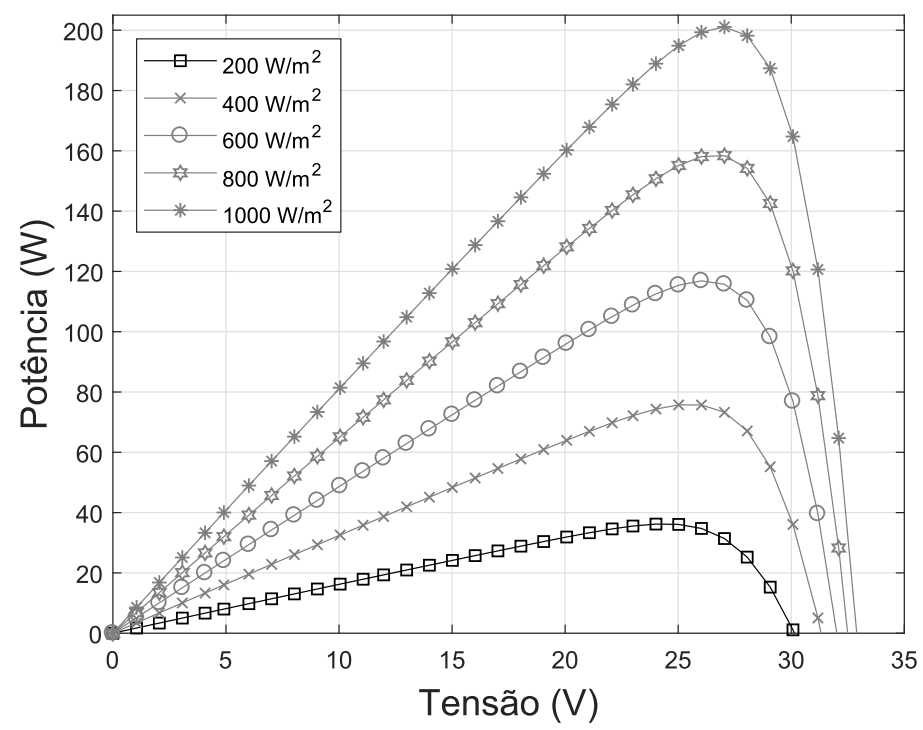

Figura 2: Curva P-V do painel Kyocera KC200GT submetido a diferentes irradiâncias e temperatura de operação igual a $25^{\circ} \mathrm{C}$.

Existem vários métodos de rastreamento do ponto de máxima potência propostos na literatura. As principais características desejadas nestes algoritmos são: rápida resposta dinâmica, erro pequeno em regime permanente, robustez a distúrbios, alta eficiência em diferentes níveis de potência. Neste trabalho são avaliados quatro algoritmos de MPPT conhecidos na literatura em diferentes cenários doravante descritos, sendo eles:

1. Fração da tensão de circuito-aberto [14];

2. Algoritmo Perturbe e Observe (P\&O) [11];

3. Algoritmo de Condutância Incremental [4];

4. Algoritmo de Condutância Incremental com passo variável [8] ;

Neste trabalho, aprimoramentos para o algoritmo apresentado em [8] são propostos, avaliando seu desempenho comparado ao dos outros quatro algoritmos supracitados.

\subsection{Fração da tensão de circuito-aberto}

Este método consiste em fixar a tensão do painel fotovoltaico em um valor calculado a partir da tensão de circuito aberto. De acordo com [3], é possível escrever a tensão de máxima potência $\left(V_{m p}\right)$ em função da tensão de circuito aberto $\left(V_{o c}\right)$, conforme a Equação (3.1).

$$
V_{m p}=V_{o c} \cdot k_{v}
$$


onde $k_{v}$ é a constante de proporcionalidade que depende das características do dispositivo fotovoltaico. Normalmente esta constante possui valor entre $71 \%$ e $76 \%$ [3].

\subsection{Algoritmo Perturbe e Observe}

Este método consiste em perturbar a tensão do painel fotovoltaico periodicamente, ou seja, aumentar ou decrementar este valor, e verificar o comportamento da potência. Se houver um aumento de potência, deve-se manter a perturbação no mesmo sentido, caso contrário, inverte-se o sentido da perturbação. Quando o ponto de máxima potência é alcançado, a tensão do painel oscila continuamente em torno deste ponto [11].

\subsection{Algoritmo de Condutância Incremental}

O Algoritmo de Condutância Incremental baseia-se no cálculo da derivada da curva $P-V$ do painel fotovoltaico. Quando o valor da derivada é positivo, significa que o ponto de operação encontra-se na subida da curva. Por outro lado, quando a derivada é negativa, o ponto de operação encontra-se na descida da curva. O ponto de máxima potência é alcançado quando a derivada é nula [14].

O sistema tenta, portanto, encontrar o ponto na curva de potência onde $\frac{d P}{d V}=0$. Desenvolvendo esta expressão, tem-se:

$$
\frac{d P}{d V}=\frac{d(V \cdot I)}{d V}=I+V \cdot \frac{d I}{d V} \approx I+V \cdot \frac{\Delta I}{\Delta V}=0
$$

Portanto, é possível perceber que:

- Caso $I+V \cdot \frac{\Delta I}{\Delta V}=0$, o ponto de operação encontra-se no ponto de máxima potência;

- Caso $I+V \cdot \frac{\Delta I}{\Delta V}>0$, o ponto de operação está à esquerda do ponto de máxima potência;

- Caso $I+V \cdot \frac{\Delta I}{\Delta V}<0$, o ponto de operação está à direita do ponto de máxima potência;

Baseado na análise anterior, toma-se a decisão de incrementar ou decrementar o valor da tensão do dispositivo fotovoltaico.

Na proposta original de [4], o tamanho do passo, isto é, o quanto se aumenta ou diminui a tensão de referência $V_{r e f}$, é fixo e pré-determinado. Atualmente alguns trabalhos mostram que tornar esse passo adaptativo pode melhorar o desempenho do algoritmo [8].

\section{ALGORITMO MPPT CONSIDERANDO MINIMIZAÇÃO DE ERRO E DERIVADA DA POTÊNCIA}

Neste trabalho, considera-se aplicação dos algoritmos de MPPT em um sistema composto por painel fotovoltaico, conversor cc-cc buck e uma bateria de 12 V. Várias simulações deste sistema 
foram realizadas onde tem-se o painel fotovoltaico conectado ao conversor buck e este à bateria. A bateria mantém a tensão de saída do conversor buck em $12 \mathrm{~V}$, enquanto que a alteração do ciclo de trabalho deste conversor varia a tensão de entrada, provida pelo painel fotovoltaico. Ou seja, o algoritmo de MPPT altera a tensão de saída do painel fotovoltaico, atuando assim no ponto de operação da curva P-V.

Regulando o ciclo de trabalho do conversor cc-cc adequadamente por meio de um algoritmo de MPPT é possível aumentar a eficiência no carregamento da bateria.

Neste trabalho, propõe-se aprimorar a atualização do passo para o algoritmo condutância incremental de modo que este apresente uma melhora em seu desempenho. Em [8], a atualização do passo é dada conforme a seguinte equação:

$$
V(k)=V(k-1) \pm N \cdot\left|\frac{\Delta P}{\Delta V-\Delta I}\right|
$$

onde $V(k)$ é tensão na saída do painel fotovoltaico regulada pelo ciclo de trabalho do conversor cc-cc no instante $k ; V(k-1)$ é tensão no instante $k-1 ; N$ é um fator de escala; $\Delta I, \Delta V$ e $\Delta P$ são as derivadas da corrente, tensão e potência de saída do painel fotovoltaico. Os valores de $\Delta I, \Delta V$ e $\Delta P$ são dados pela Equações (4.2), (4.3) e (4.4).

$$
\begin{gathered}
\Delta I=I(k)-I(k-1) \\
\Delta V=V(k)-V(k-1) \\
\Delta P=P(k)-P(k-1)
\end{gathered}
$$

De acordo com a Equação (3.2), nota-se que quanto mais próximo do ponto de máxima potência, menor será o valor de $I+V \cdot \frac{\Delta I}{\Delta V}$, uma vez que esta expressão representa a derivada da curva de potência em relação à tensão. Manipulando esta expressão e a igualando a zero, obtém-se:

$$
I+V \cdot \frac{\Delta I}{\Delta V}=0 \rightarrow-\frac{I}{V}=\frac{\Delta I}{\Delta V} \rightarrow \frac{I}{V}+\frac{\Delta I}{\Delta V}=0,
$$

que é válida apenas no ponto de máxima potência. Em outros pontos, a soma do lado esquerdo da Equação 4.5 não será igual a zero. Esta diferença entre o valor obtido e o desejado (zero), representa o erro em relação ao ponto de máxima potência, e pode então ser dado pela seguinte equação:

$$
\text { erro }=\frac{I}{V}+\frac{\Delta I}{\Delta V}
$$

Minimizando este erro, é possível encontrar o ponto de máxima potência.

Observando a curva $\mathrm{P}-\mathrm{V}$ de um painel fotovoltaico, nota-se que quanto mais próximo do ponto de máxima potência, menor deve ser o valor do passo da tensão para alcançá-lo. Portanto, neste artigo se propõe que o valor do passo dado na curva $P-V$ seja dependente do erro dado pela Equação (4.6). Além disso, para aumentar a velocidade de convergência e diminuir as oscilações em torno do ponto de máxima de potência, utiliza-se também a derivada da potência no tempo, dada pela 
Equação (4.4). Desta forma, a proposta de atualização do passo deste trabalho é representada pela seguinte equação:

$$
V(k)=V(k-1) \pm N \cdot \mid \Delta P \cdot \text { erro } \mid
$$

onde $N$ é um fator de escala e foi ajustado manualmente. Foram testados vários valores entre 0,01 e 1 , e o que apresentou os melhores resultados foi $N=0,15$.

Observando a Equação (4.7), nota-se que quanto mais distante do ponto de máxima de potência, maior será o erro, e portanto maior será o passo de tensão dado pelo algoritmo. A medida que se aproxima do ponto de máxima potência, o erro diminui, e os passos do algoritmo se tornam cada vez menores. Além disso, o termo $\Delta P$ provê uma maior velocidade de convergência para o algoritmo, principalmente em mudanças abruptas das condições ambientais, como irradiância e temperatura. Para que o tamanho do passo do algoritmo não seja demasiadamente grande, foi definida uma saturação no valor do passo, de modo que a maior alteração de tensão entre uma iteração $k$ e $k+1$ seja de $2 V$. O Algoritmo 1 apresenta a proposta deste trabalho para seguimento do ponto de máxima potência. Os parâmetros $\delta V, \delta I, \delta P$ são valores próximos de zero.

\subsection{Metodologia de Avaliação dos Algoritmos}

Com o objetivo de simular o desempenho do algoritmo proposto, alguns cenários foram definidos. O primeiro cenário possui irradiância e temperatura fixas, com seus valores dados conforme as Condições Padrões de Teste para os painéis fotovoltaicos, $1000 \mathrm{~W} / \mathrm{m}^{2}$ e $25^{\circ} \mathrm{C}$, respectivamente. $\mathrm{O}$ segundo cenário de teste possui valores de irradiância e temperatura reais, medidos de janeiro a dezembro de 2017. Os dados reais de irradiância e temperatura foram cedidos pelo Projeto de Pesquisa e Desenvolvimento (P\&D) com o título "Instalação de um Sistema de Geração Solar Fotovoltaica de $34 \mathrm{kWp}$ em telhado de um prédio da Escola de Engenharia Elétrica, Mecânica e de Computação (EMC) Universidade Federal de Goiás (UFG)”, em Goiânia - GO.

As avaliações dos algoritmos de MPPT são realizadas por meio dos seguintes parâmetros: Raiz Quadrada do Erro Quadrático Médio (REQM), a eficiência do algoritmo em relação a energia produzida, e a energia gerada. A REQM é dada pela Equação (4.8), onde $P_{\text {painel }}(t)$ é a potência instantânea do painel encontrada pelo algoritmo MPPT, e $P_{\text {ideal }}(t)$ é a máxima potência que o painel poderia fornecer para aquela situação de irradiância e temperatura, e $m$ é numero de amostras. A $P_{\text {ideal }}(t)$ foi estimada por meio de uma varredura da curva P-V.

$$
R E Q M=\sqrt{\frac{1}{m} \sum_{1}^{m}\left(P_{\text {painel }}-P_{\text {ideal }}\right)^{2}}
$$

A eficiência é dada pela razão entre a energia produzida pelo painel e a máxima energia que este poderia produzir caso estivesse sempre operando no ponto de máxima de potência. A eficiência é dada pela seguinte equação:

$$
\eta=\frac{\int_{t 1}^{t 2} P_{\text {painel }}(t) \mathrm{d} t}{\int_{t 1}^{t 2} P_{\text {ideal }}(t) \mathrm{d} t}
$$




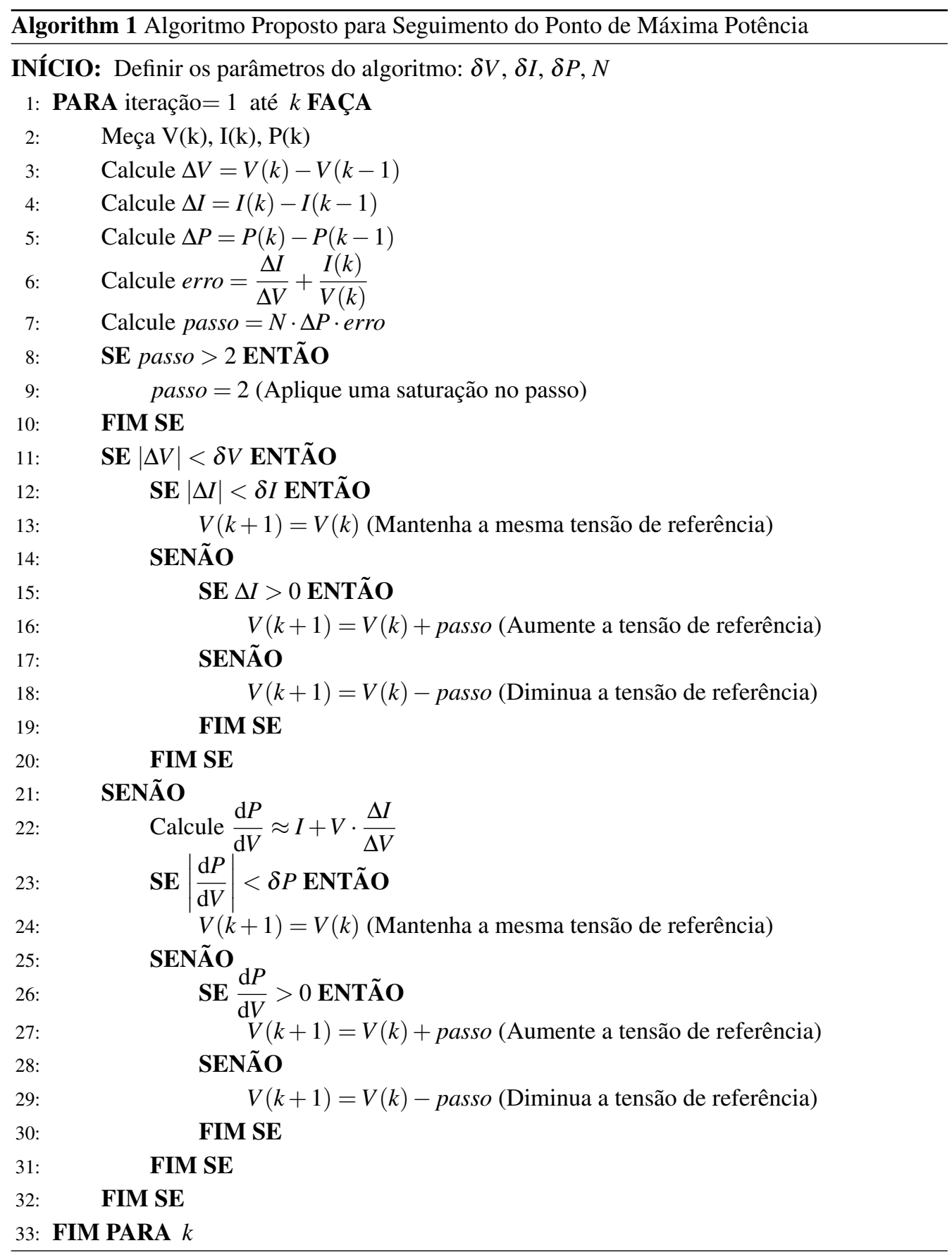


A energia produzida pelo painel é calculada de acordo com:

$$
\text { Energia }=\int_{t 1}^{t 2} P_{\text {painel }}(t) \mathrm{d} t
$$

\section{SIMULAÇÕES E RESULTADOS}

Conforme mencionado na Seção 4, dois casos de simulação são considerados para análise de desempenho dos algoritmos de MPPT: irradiância e temperatura fixas e variáveis (medidas reais). Em [7], é realizada a estimação dos parâmetros do modelo de 1 diodo para irradiância de 1000 $W / m^{2}$ e temperatura do painel de $25^{\circ} C$, e posteriormente sua adaptação em função da irradiância e temperatura. Os valores obtidos por [11] foram utilizados neste trabalho, e têm como valor: $n=1,2277 ; R_{s}=0,1477 \Omega ; R_{s h}=92,31 \Omega ; I_{0}=32,23 \mu A$ e $\operatorname{Iir} r=8,223 A$. A adaptação dos parâmetros em função das condições ambientais de irradiância e temperatura também é realizada neste trabalho, conforme feito em [7].

\subsection{Irradiância e temperatura fixas}

Nesta seção, são apresentados os resultados para o caso de irradiância e temperatura fixas. É possível analisar as velocidades de convergência de cada algoritmo e o seu erro em regime permanente. A Figura 3 apresenta uma comparação das potências de saída do painel fotovoltaico para cada algoritmo. A convergência do algoritmo Fração da Tensão de Circuito Aberto é rápida quando comparada com os outros algoritmos deste trabalho, porém há alguns fatores negativos. $\mathrm{O}$ valor da constante $k_{v}$ deve ser ajustado manualmente, e esta constante muda para cada tipo de painel. Os desempenhos dos algoritmos Perturbe e Observe e Condutância Incremental, com a irradiância constante, são semelhantes tanto no quesito convergência quanto no valor final. Por outro lado, o algoritmo de Condutância Incremental com o passo variável proposto por [8] apresenta uma convergência mais rápida que no caso com o passo fixo. Em sua $8^{\mathrm{a}}$ iteração, a potência de saída do painel atinge o valor ideal, enquanto que no caso com o passo fixo isto ocorre apenas na $12^{\mathrm{a}}$ iteração. Além disso, com o passo variável a diferença nos valores de tensão são cada vez menores. Com o algoritmo proposto neste trabalho, nota-se que em apenas 5 iterações a potência de saída do painel atingiu seu valor ideal, sem apresentar oscilações de tensão.

A Tabela 1 apresenta uma comparação dos 5 algoritmos MPPT nos quesitos REQM, Eficiência, e Energia Gerada. Observa-se que o algoritmo proposto possui o menor REQM, e os maiores valores de eficiência e energia produzida.

A Figura 4 mostra o REQM instantâneo em cada iteração. É interessante perceber que o algoritmo proposto obtém seu menor erro na iteração 5, enquanto que o Condutância Incremental com passo variável proposto por [8] necessita de mais de 30 iterações para isto. Portanto, o algoritmo proposto apresenta velocidade de convergência maior do que as dos algoritmos Condutância Incremental e Perturbe e Observe, de tal forma que a soma dos erros acumulados fica menor do que de todos os algoritmos considerados, conforme mostrado na Tabela 1. 


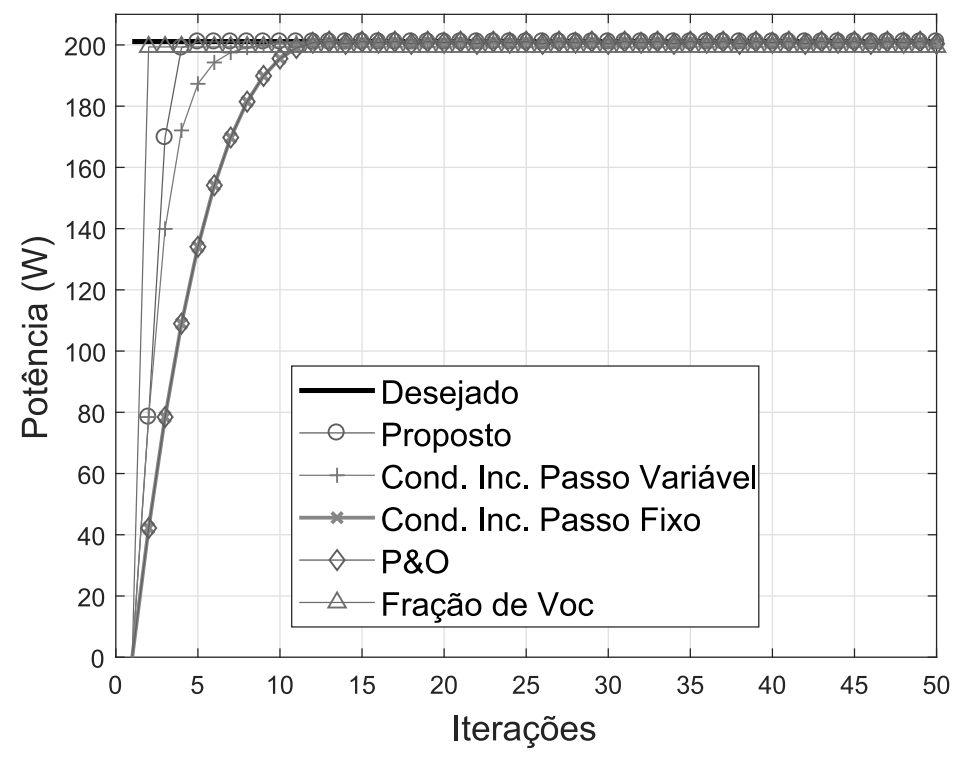

Figura 3: Comparação da potência do painel fotovoltaico utilizando diferentes algoritmos de MPPT para Irradiância de $1000 \mathrm{~W} / \mathrm{m}^{2}$ e Temperatura de $25^{\circ}$

Tabela 1: Comparação dos algoritmos considerando irradiância e temperatura fixas

\begin{tabular}{cccc}
\hline Algoritmo & REQM & Eficiência & Energia $(\mathbf{W h )}$ \\
\hline Proposto & 33,6122 & 0,9641 & 161.5705 \\
\hline Fração da de $V_{o c}$ & 33,8858 & 0,9553 & 160,0935 \\
\hline Perturbe e Observe & 44,1553 & 0,9234 & 154,7556 \\
\hline Cond. Inc. & 44,1553 & 0,9234 & 154,7556 \\
\hline Loukriz et al. [8] & 34,7403 & 0,9559 & 160,2080 \\
\hline
\end{tabular}

\subsection{Irradiância e temperatura reais}

No cenário considerado nesta seção, os algoritmos de MPPT são analisados com a irradiância e temperatura reais, medidas no painel fotovoltaico Kyocera KC200GT [5], localizado na cidade de Goiânia - GO. Os dados foram coletados de $1^{\circ}$ janeiro a 31 dezembro de 2017. A Figura 5 apresenta os valores de irradiância e temperatura medidas no painel fotovoltaico no ano de 2017. O tempo de amostragem dos valores foi de 1 em 1 minuto.

A Figura 6 apresenta uma comparação da energia gerada mensalmente no ano de 2017 pelo painel fotovoltaico utilizando o algoritmo proposto, o algoritmo de [8] e o valor máximo. Observa-se que durante os 12 meses a curva do algoritmo proposto ficou mais próxima do valor desejado do 


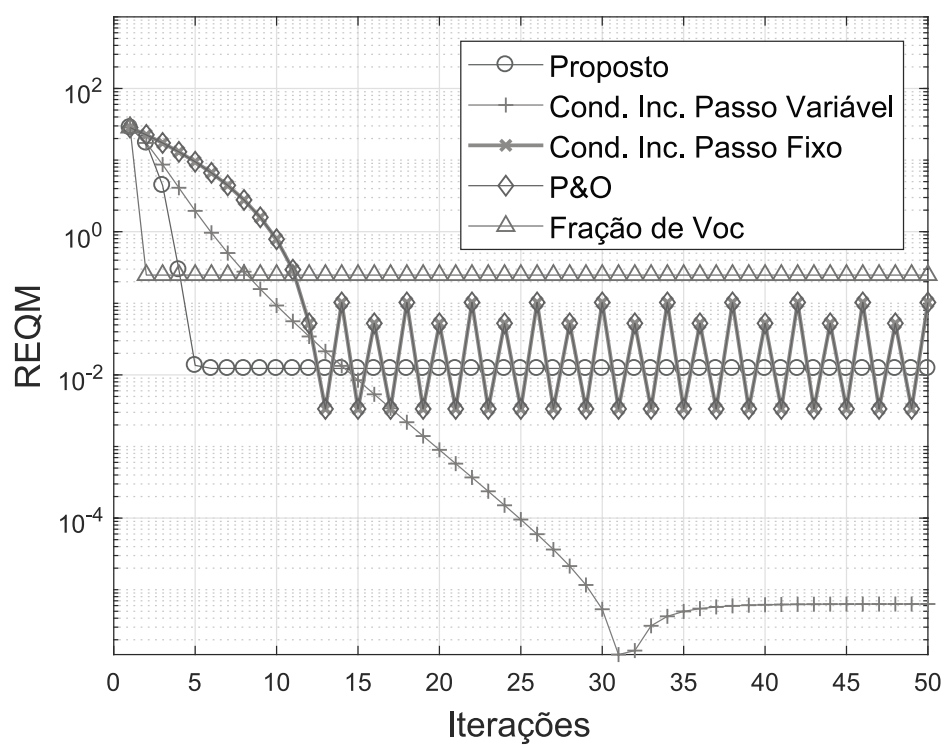

Figura 4: Comparação da convergência da raiz quadrada do erro quadrático médio para diferentes algoritmos de MPPT para Irradiância e Temperatura constantes
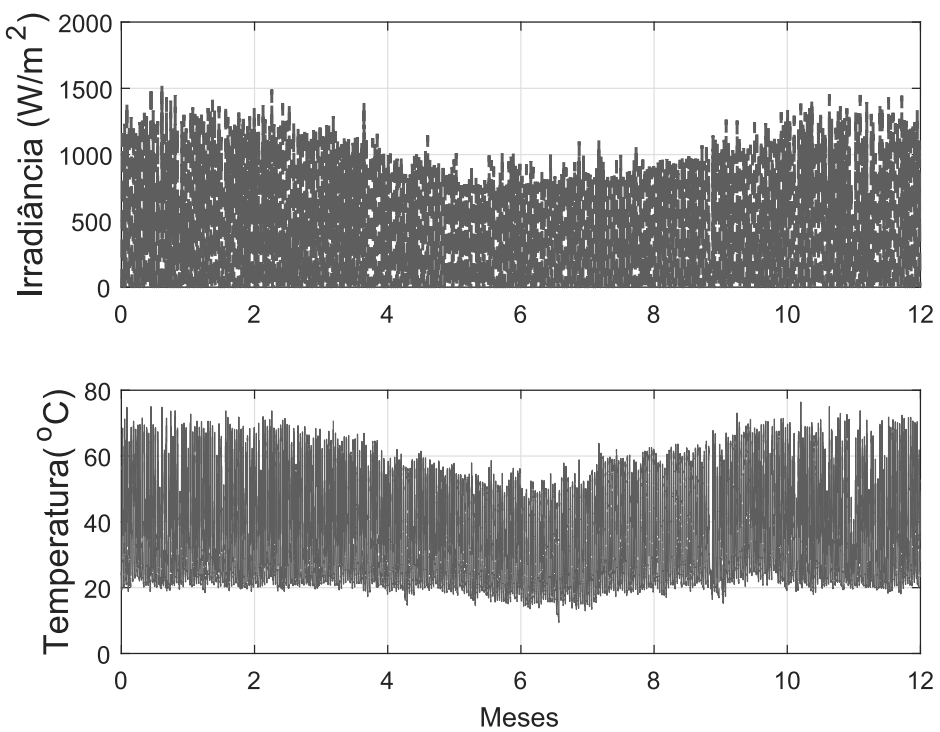

Figura 5: Irradiância e Temperatura medidas no painel fotovoltaico no ano 2017. 


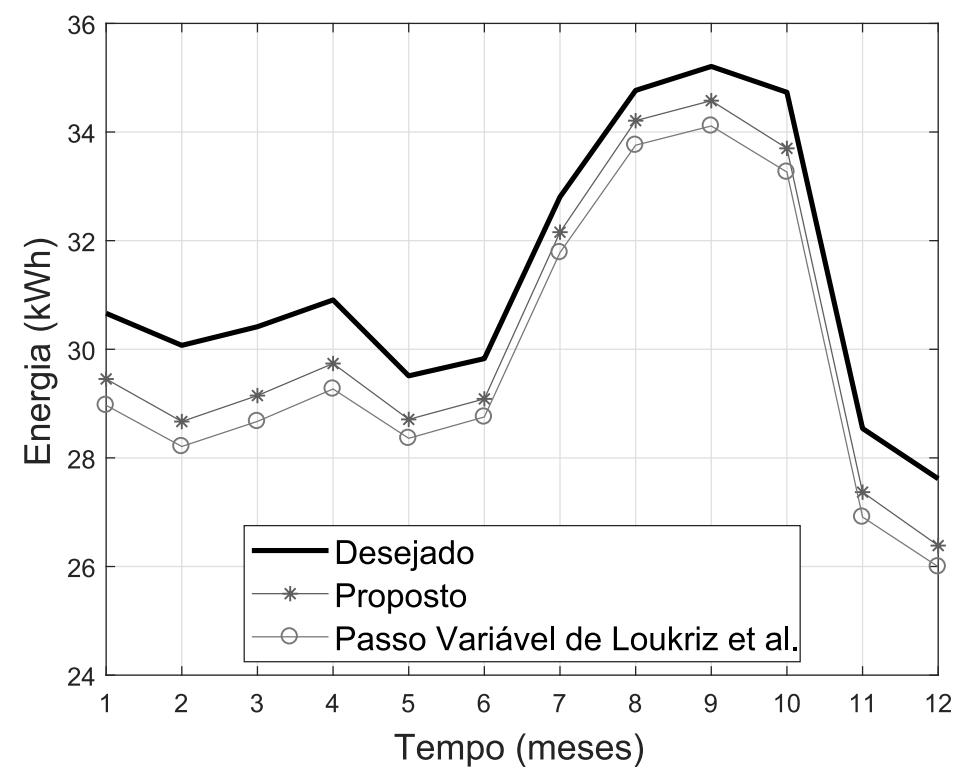

Figura 6: Comparação da energia gerada por mês utilizando cada algoritmo.

que a curva do algoritmo de [8]. Portanto, utilizando o algoritmo proposto é possível gerar mais energia.

A Tabela 2 apresenta uma comparação dos desempenhos entre os algoritmos para o caso com irradiância e temperatura reais.

Tabela 2: Comparação dos algoritmos no caso com irradiância e temperatura reais medidas no ano de 2017.

\begin{tabular}{cccc}
\hline Algoritmo & REQM & Eficiência & Energia $(\mathbf{W h})$ \\
\hline Proposto & 9,2577 & 0,9684 & $3,6322 \cdot 10^{5}$ \\
\hline Fração da de $V_{o c}$ & 14,5296 & 0,8255 & $3,0687 \cdot 10^{5}$ \\
\hline Perturbe e Observe & 12,9847 & 0,8850 & $3,3271 \cdot 10^{5}$ \\
\hline Cond. Inc. & 12,1254 & 0,8996 & $3,3745 \cdot 10^{5}$ \\
\hline Loukriz et al. [8] & 11,3087 & 0,9546 & $3,5807 \cdot 10^{5}$ \\
\hline
\end{tabular}

O algoritmo proposto apresentou a melhor eficiência, 96,84\%, produzindo desta forma a maior quantidade de energia, 363,22 $\mathrm{kWh}$. Observando o REQM, nota-se que o algoritmo proposto apresentou o menor erro entre todos os analisados. $\mathrm{O}$ algoritmo de [8] apresenta uma eficiência menor do que a do algoritmo proposto, 95,46\% contra 96,84\%. Entretanto, esta diferença se torna mais notável quando se observa a energia gerada. Com o algoritmo proposto foi possível gerar mais de $363 \mathrm{kWh}$, enquanto que com o de [8] apenas $358 \mathrm{kWh}$. Os resultados revelam que 
é possível afirmar que ao longo do ano de 2017, na média, o desempenho do algoritmo proposto foi superior ao dos demais comparados neste trabalho.

\title{
6 CONCLUSÕES
}

Este trabalho apresentou uma proposta de atualização do passo do algoritmo condutância incremental, considerando o mesmo variável. Nos dois cenários analisados, irradiância e temperatura fixas e reais, o algoritmo proposto mostrou um desempenho superior, apresentando uma convergência mais rápida e erros quadráticos médios menores do que os outros algoritmos considerados. Esta eficiência afeta diretamente a produção de energia do painel fotovoltaico. Um algoritmo mais eficiente, como o proposto neste trabalho, faz com que o painel produza mais energia, o que justifica a sua implementação.

\section{AGRADECIMENTOS}

O presente trabalho foi realizado com apoio da Coordenação de Aperfeiçoamento de Pessoal de Nível Superior - Brasil (CAPES) - Código de Financiamento 001.

\begin{abstract}
In this work, a new maximum power point tracking algorithm (MPPT) for photovoltaic systems is proposed. The considered system is composed by a photovoltaic panel charging a battery using a buck-type dc-dc converter. Computational simulations are carried out to compare the performance of the MPPT algorithms, analyzing the output voltage and output power behavior at the photovoltaic panel. Three evaluation scenarios were considered: with constant irradiance and temperature, randomly chosen and using real data. The results of the simulations show that the proposed algorithm provides better performance for the photovoltaic system than the main MPPT algorithms in the literature.
\end{abstract}

Keywords: MPPT algorithm, solar energy, photovoltaic panel.

\section{REFERÊNCIAS}

[1] E.P. Carvalho, J.B. Gonçaves \& P.P.L. Prado. A New Approach for Maximum Power Point Tracking. 21st Brazilian Congress of Mechanical Engineering, (2011).

[2] E.N. Chaves, J.H. Reis, E.A.A. Coelho, L.C.G. de Freitas, J.B.V. Junior \& L.C. Freitas. Simulated Annealing-MPPT in Partially Shaded PV Systems. IEEE Latin America Transactions, 14(1) (2016), 235-241.

[3] T. Esram \& P.L. Chapman. Comparison of photovoltaic array maximum power point tracking techniques. IEEE Transactions on energy conversion, 22(2) (2007), 439-449.

[4] K. Hussein, I. Muta, T. Hoshino \& M. Osakada. Maximum photovoltaic power tracking: an algorithm for rapidly changing atmospheric conditions. IEE Proceedings-Generation, Transmission and Distribution, 142(1) (1995), 59-64. 
[5] Kyocera. "High efficiency multicrystal photovoltaic module KC200GT (Datasheet)" (n.d.). URL https://www.kyocerasolar.com/dealers/product-center/archives/spec-sheets/ KC200GT.pdf.

[6] M.C.C. Leite, F.A.M. Vieira, V.B. Silva, M.Z. Fortes \& D.H.N. Dias. Harmonic Analysis of a Photovoltaic Systems Connected to Low Voltage Grid. IEEE Latin America Transactions, 16(1) (2018), $112-117$.

[7] G. Lopes Filho, R.A.P. Franco \& F.H.T. Vieira. Estimação de parâmetros de painéis fotovoltaicos utilizando o algoritmo firefly com atualização em função da irradiância e temperatura. In "VII Congresso Brasileiro de Energia Solar-CBENS 2018” (2018).

[8] A. Loukriz, M. Haddadi \& S. Messalti. Simulation and experimental design of a new advanced variable step size Incremental Conductance MPPT algorithm for PV systems. ISA transactions, 62 (2016), $30-38$.

[9] MathWorks. "Softwares de simulação: Matlab e Simulink" (n.d.). URL https : //www . mathworks . com/.

[10] N.E.M. Moçambique, R.Q. Machado \& V.A. Oliveira. A fuzzy PD-PI control strategy to track the voltage references of photovoltaic arrays. In "2011 9th IEEE International Conference on Control and Automation (ICCA)” (2011), pp. 1162-1167. doi:10.1109/ICCA.2011.6138046.

[11] N.E.M. Moçambique. "Aplicação de Algoritmos de Busca do Ponto de Máxima Potência e controladores lineares e/ou Fuzzy para a regulação da tensão terminal de Painéis Fotovoltaicos”. Ph.D. thesis, Universidade de São Paulo (2012).

[12] A. Pallavee Bhatnagar \& B. Nema. Conventional and global maximum power point tracking techniques in photovoltaic applications: A review. Journal of Renewable and Sustainable Energy, 5(3) (2013), 032701.

[13] M.G. Villalva, J.R. Gazoli \& E.R. Filho. Comprehensive Approach to Modeling and Simulation of Photovoltaic Arrays. IEEE Transactions on Power Electronics, 24(5) (2009), 1198-1208. doi:10.1109/ TPEL.2009.2013862.

[14] M.G. Villalva et al. "Conversor eletrônico de potência trifásico para sistema fotovoltaico conectado à rede elétrica” (2010). 